\title{
Core outcome set measurement for future clinical trials in acute myeloid leukemia: the HARMONY study protocol using a multi- stakeholder consensus-based Delphi process and a final consensus meeting
}

Katharina M. Lang ${ }^{1 *}$ D, Kathryn L. Harrison ${ }^{2}$, Paula R. Williamson ${ }^{3}$, Brian J. P. Huntly ${ }^{4}$, Gert Ossenkoppele ${ }^{5}$, Jan Geissler ${ }^{6}$, Tamàs Bereczky ${ }^{6}$, Jesús M. Hernández-Rivas ${ }^{7}$, Hélène Chevrou-Séverac ${ }^{8}$, Rory Goodbody ${ }^{9}$, Renate Schulze-Rath ${ }^{10}$ and Lars Bullinger ${ }^{1}$

\begin{abstract}
Background: Acute myeloid leukemia (AML) is the most common acute leukemia in adults and has an unacceptably low cure rate. In recent years, a number of new treatment strategies and compounds were developed for the treatment of AML. There were several randomized controlled clinical trials with the objective to improve patients' management and patients' outcome in AML. Unfortunately, these trials are not always directly comparable since they do not measure the same outcomes, and currently there are no core outcome sets that can be used to guide outcome selection and harmonization in this disease area. The HARMONY (Healthcare Alliance for Resourceful Medicine Offensive against Neoplasms in Hematology) Alliance is a public-private European network established in 2017 and currently includes 53 partners and 32 associated members from 22 countries. Amongst many other goals of the HARMONY Alliance, Work Package 2 focuses on defining outcomes that are relevant to each hematological malignancy. Accordingly, this pilot study will be performed to define a core outcome set in AML.
\end{abstract}

Methods: The pilot study will use a three-round Delphi survey and a final consensus meeting to define a core outcome set. Participants will be recruited from different stakeholder groups, including patients, clinicians, regulators and members of the European Federation of Pharmaceutical Industries and Associations. At the preDelphi stage, a literature research was conducted followed by several semi-structured interviews of clinical public and private key opinion leaders. Subsequently, the preliminary outcome list was discussed in several multistakeholder face-to-face meetings. The Delphi survey will reduce the preliminary outcome list to essential core outcomes. After completion of the last Delphi round, a final face-to-face meeting is planned to achieve consensus about the core outcome set in AML.

(Continued on next page)

\footnotetext{
* Correspondence: katy.harrison@nice.org.uk

${ }^{1}$ Charité University Medicine, Chariteplatz 1, Berlin 10117, Germany

Full list of author information is available at the end of the article
} 
(Continued from previous page)

Discussion: As part of the HARMONY Alliance, the pilot Delphi aims to define a core outcome set in AML on the basis of a multi-stakeholder consensus. Such a core outcome set will help to allow consistent comparison of future clinical trials and real-world evidence research and ensures that appropriate outcomes valued by a range of stakeholders are measured within future trials.

Keywords: Delphi process, Core outcome set, Acute myeloid leukemia

\section{Introduction}

Acute myeloid leukemia (AML) is the most common acute leukemia in adults. Clonal expansion of undifferentiated myeloid precursor cells causes impaired hematopoiesis and bone marrow failure. For decades, the basis of AML treatment has remained virtually unchanged. However, over the last few years, driver mutations have been identified and molecular subgroups defined, resulting in an improved prognostic stratification and updated European LeukemiaNET guidelines [1, 2].

Although scientific and technical advances have resulted in a number of new treatment strategies in recent years, AML still poses a challenge to curative approaches: cure rates remain poor compared with those of other hematological malignancies. Currently, several innovative compounds are being investigated in randomized controlled clinical trials with the objective to improve both patients' outcome and patients' management in AML. However, the ability to compare these clinical trials is limited by differences in their measured outcomes. This lack of standardization relates to the current lack of a core outcome set (COS) that can be used to guide outcome selection and harmonization in AML in current and future trials.

The HARMONY (Healthcare Alliance for Resourceful Medicine Offensive against Neoplasms in Hematology) Alliance is a public-private European network established in 2017 and currently includes 53 partners, inter alia six cancer patient umbrella organizations, and 32 associated members from 22 countries. One of HARMONY's goal is to use big data to improve understanding and treatment of hematological malignancies. In order to achieve this aim, HARMONY is structured into eight work packages, of which Work Package 2 (WP 2) is focused on defining outcomes that are relevant to each hematological malignancy. In the future, WP 2 aims to define a common COS valid for all hematological malignancy. Accordingly, this pilot study will be performed to define a COS in AML.

A COS is a minimum set of outcomes developed by consensus, usually using a multi-stakeholder consensusbased Delphi process. The COS is a reference point and provides the minimum outcome set that should be collected in further clinical trials on a given condition. Use of a COS improves comparability of clinical trials and consistency of reporting, reduces selective reporting bias and ensures that appropriate outcomes valuable by a range of stakeholders are measured. Furthermore, a COS can be used in other clinical settings or type of realworld evidence research and can be incorporated into clinical guidelines and improve clinical practice in this way.

Involvement of different stakeholder groups is critical to ensure that the defined COS has broad relevance. Key stakeholders will provide their expert feedback and will be recruited on the basis of their experiences relevant to AML or the project. The different stakeholder groups include health service users, health service practitioners, researchers, drug developers, regulators, patient advocates and patients. All ratings are provided anonymously; so without group interactions, different opinions will be presented more clearly between different stakeholder groups.

\section{Aims}

This project aims to define a COS in AML agreed by consensus of all stakeholder groups to be measured in future clinical trials and observational studies. This pilot study also aims to establish the Delphi method for further disease-specific COS defining studies that are planned for other hematological malignancies in HARMONY. The defined COS will help to improve future clinical study design to improve patient satisfaction and management.

\section{Methods}

COS development will follow recommendations of the Core Outcome Measures in Effectiveness Trials (COMET) initiative from the international Core Outcome Set Standards for Development (COS-STAD) [3, 4]. The Delphi method will be used to achieve a consensus from different stakeholder groups. A prospective study protocol was registered in the COMET database (http://www.comet-initiative.org/studies/details/1347). The protocol was written in accordance with the Core Outcome Set-Standardised Protocol (COS-STAP) recommendations [5] in cooperation between WP 2 and WP 6. In Fig. 1, each step of the study is illustrated. 


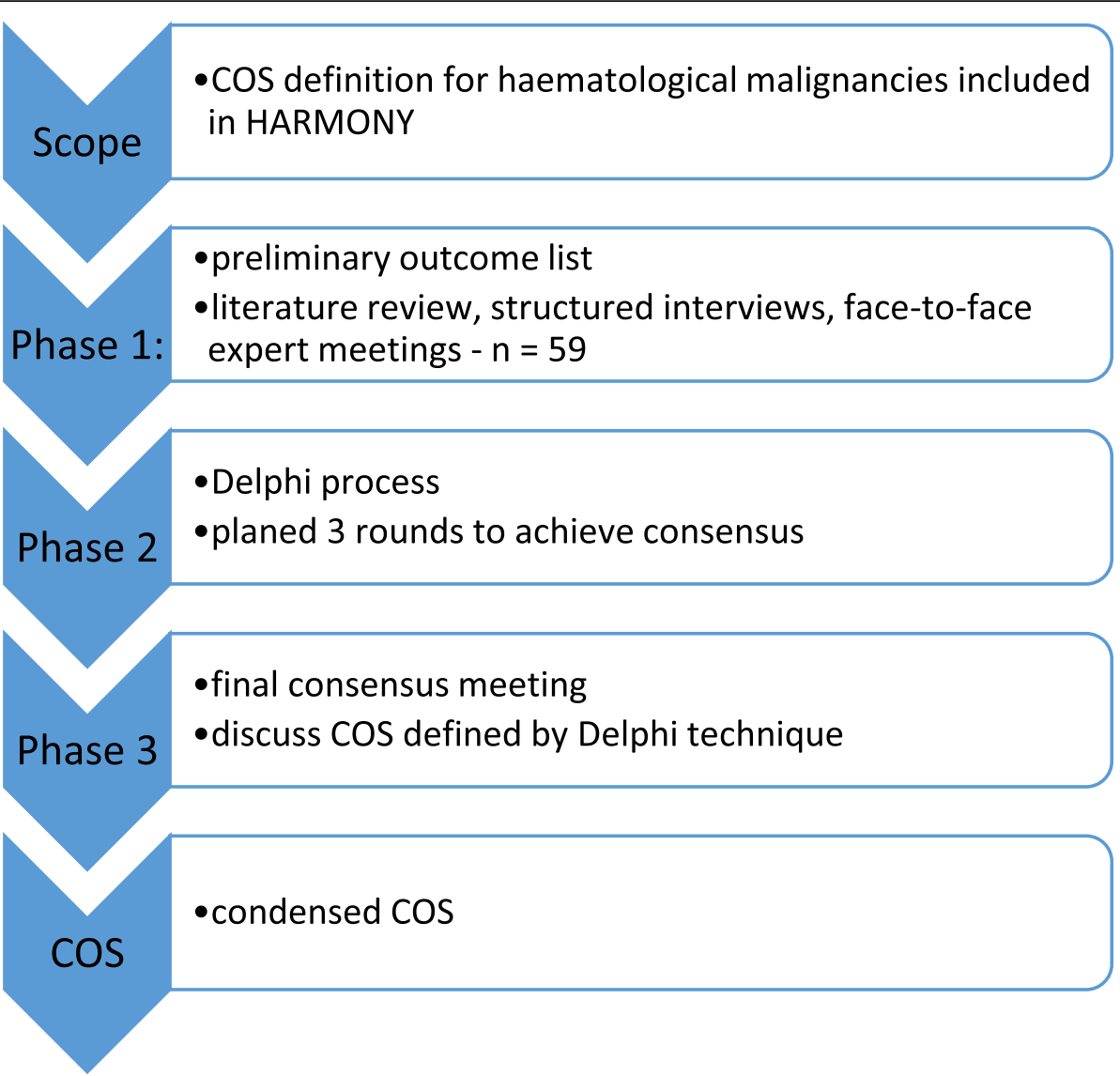

Fig. 1 Steps of the planned Delphi process

\section{Study design}

Recruitment of participants mainly takes place from members of the HARMONY WPs, but participants outside the HARMONY Alliance are also invited to take part in the Delphi survey within their stakeholder group. At least three rounds of the Delphi survey are planned to achieve consensus. At the end, a final face-to-face consensus meeting will take place.

\section{Scope of the COS}

The COS-identified AML will be crucial not only for the HARMONY project but for all future analyses. As the definition of COS will determine what data are captured for AML, it will also impact how the analyses will be available in the future. Consequently, COS will directly impact future data sets available to HARMONY and also the research community. Additionally, future work conducted by HARMONY partners and other stakeholders in the AML field will be directly impacted by the defined COS.

\section{Participants}

1. Patients
Every patient older than 18 years with AML can participate. Different subtypes of AML are equally included, regardless of previous treatments, including stem cell transplantation. Patients treated as outpatients were included as well as patients treated in hospitals. Owing to the use of English for the Delphi survey, participation is limited to patients understanding English.

2. Clinicians and clinical researchers

Every clinician within or outside the HARMONY

Alliance who has experience in AML treatment can participate.

3. Drug developers

Members of European Federation of Pharmaceutical Industries and Associations (EFPIA) and part of the HARMONY Alliance are invited to participate as well.

4. Regulators

Recruitment of participants will be performed within the HARMONY Alliance with the support of WP 7, which is responsible for dissemination, communication and training within the HARMONY Alliance. WP 6 provides assistance in promotion for the survey. 
To recruit health-care professionals, AML key opinion leaders will be contacted and will be asked to invite other professionals within their peer groups to take part in the Delphi survey. Thus, participants outside the HARMONY Alliance are welcome to take part in the Delphi survey. Patient recruitment will be performed with the support of patient advocates and several patient organizations, like Acute Leukemia Advocates Network or LeukaNet. Especially in the recruitment of patients, social networks and internal communication tools will be used to spread the information and invitations for the Delphi survey. In the next rounds, personal mail reminders will be sent out to enhance the response rates.

\section{Trial registration}

This project has been registered (May 2019) in the database of the COMET initiative

(http://www.comet-initiative.org/studies/details/1347).

\section{Study management group}

As recommended by the COMET initiative, a study management group has been assembled to oversee the project [3]. The group comprises a study coordinator, a hematologist with leading roles in AML treatment and clinical trials, a drug developer with experience in past and current trials, patient advocates and methodological experts with experience of systematic reviews and Delphi studies. The role of the study management group is to support the development of the study protocol and to review the list of outcomes and the associated lay versions and descriptions.

\section{Selection of the preliminary outcome list for AML}

So far, the empirical basis for identifying a long list of preliminary outcomes relevant in AML for the Delphi study has been threefold:

1. A literature research was conducted in the COMET database to get an overview of the cancer outcomes already used in existing clinical trials. Therefore, all relevant trials for outcome set definition in AML were collected to October 17, 2017 [6]. The primary AML outcome list was generated by extracting outcomes from the COMET database research and review of published literature [7-14].

2. Several semi-structured interviews of clinical public and private key opinion leaders were conducted to assess the initial selection of the outcome parameters, and additional outcomes were supplemented. This was followed by several face-to-face meetings to further expand and discuss the potential outcome list, including a multi-stakeholder group workshop and a further meeting with European AML key opinion leaders within the scope of the HARMONY Alliance.

3. Patient representatives and patients were consulted to include patients' perspective. The preliminary outcome list was complemented by including additional outcomes and revising in accordance with patients' comments.

On the basis of new scientific knowledge [15] and recommendations of the COMET initiative [3], the outcome list was reworked. Prognostic factors (e.g., age and gender) were removed from the list. Tools of "how to measure" an outcome (e.g., quality-of-life questionnaires) were also removed. Instead, AML concepts (outcomes related to patients' perception of their symptoms, functioning and health-related quality of life) were included.

After the pre-Delphi stage, a list of 59 outcomes, each with a short plain language description, grouped into eight domain categories was completed. You can find the list used for this Delphi survey as Additional file 1.

\section{Delphi process}

The Delphi survey will be managed online by using DelphiManager software maintained by the COMET initiative [6]. Invitations with a registration link will be sent out. After registration, every participant will receive a unique identification code to take part in the Delphi survey. For registration, participants are asked to fill in their email address, their stakeholder group and their home country. The webpage includes a description of aims and objectives of the survey and gives an explanation about how to complete the online Delphi survey. In every round, the participants will be asked to rate the importance of each outcome on the basis of their personal experiences. Each outcome will be ranked into three categories ("not important", "important, but not critical" and "critical") by using a 9-point Likert scale.

After the first round, a descriptive statistic for every stakeholder group will be provided. Only participants who completed the first round will be invited to take part in the second Delphi round. In the following rounds, participants will revise their answers by taking the previous results into account. The process is stopped after pre-defined consensus criteria.

\section{Consensus criteria}

To reduce potential bias in interpretation of the results, a clear consensus definition is important. We will use three categories of consensus that were already used in previous works [16].

1. "Consensus in" means $70 \%$ or more of all respondents scored the outcome as critically 
important and $15 \%$ or fewer of all respondents scored the outcome as of limited importance.

2. "Consensus out" means $70 \%$ or more of all respondents scored the outcome as of limited importance and $15 \%$ or fewer of all respondents scored the outcome as critically important.

3. No consensus.

Outcomes that do not achieve consensus through several Delphi rounds will be discussed in a final face-toface consensus meeting to finally ratify the AML COS. Representatives from all participating stakeholder groups will be part of this meeting.

\section{Analysis}

Analysis of the Delphi study will use descriptive statistics. The results for each Delphi round, for each outcome and for each stakeholder group will be presented in frequency tables. The analysis of the Delphi survey will be performed by using the $\mathrm{R}$ statistical software version 3.5.2.

As an exploratory analysis, we additionally identify outcomes considered important for patients. The median Likert score for the patient group at the end of each round will be calculated, and those outcomes achieving a median of at least 7 will be considered important for patients and will be included in the COS. In this way, patient-important outcomes can be separately discussed in the final consensus meeting. Attrition bias will be investigated by comparing results across participants who complete successive rounds versus those who withdraw at round 2 or 3 . Until now, no valuable data are available about the best group size for a Delphi survey; in this AML pilot Delphi, a group size of 20-50 participants should be achieved.

\section{Discussion}

The described modified Delphi process will help to define a COS for AML on the basis of consensus of different stakeholder groups. To ensure the impact of patients' involvement, an additional criterion in analysis will mark the outcomes with special interest for patients.

The language used in this Delphi survey is English. Therefore, participation is limited to men and women with a sufficient command of English to read and understand the survey. This constitutes a restriction of the study, but translation in other European languages is not proposed for the pilot Delphi.

A further limitation of the study is recruitment of participants in the course of the HARMONY communication platform and patient umbrella organizations.

Participants will be asked about their home country at registration.
The anticipated way of COS development ensures that clinicians, EFPIA members, health authorities and patients have an equal share in each stage of the process.

Carrying out a pilot Delphi will greatly inform the design and success of further Delphi surveys for COS definition for other hematological diseases considered in HARMONY.

The defined COS should be considered a minimum set of outcomes for AML, which will be collected and reported in future clinical trials, real-world evidence research and observational studies. In addition, further outcomes of special interest that are deemed relevant can be included in future research.

After the definition of a COS in AML, the next challenge will be the implementation of these outcomes in clinical guidelines and at last in clinical practice. Finally, patient treatment and patient satisfaction can be improved by reducing heterogeneity among clinical trials. The measured outcomes will be more easily comparable, meta-analyses of pooled studies will be more meaningful, and the level of evidence of future guidelines will be improved.

\section{Trial status}

At the time of manuscript submission, the Delphi survey was open to recruitment. The starting date was April 12, 2019, and the approximate closing date was June 9, 2019. Protocol version 17 was published April 12, 2019.

\section{Supplementary information}

Supplementary information accompanies this paper at https://doi.org/10. 1186/s13063-020-04384-1.

Additional file 1. Preliminary acute myeloid leukemia (AML) outcome list.

Additional file 2. Core Outcome Set-Standardised Protocol (COS-STAP) checklist

\section{Abbreviations}

AML: Acute myeloid leukemia; COMET: Core Outcome Measures in Effectiveness Trials; COS: Core outcome set; EFPIA: European Federation of Pharmaceutical Industries and Associations; HARMONY: Healthcare Alliance for Resourceful Medicine Offensive against Neoplasms in Hematology; WP: Work Package

\section{Acknowledgements}

We thank Eleni Vradi and Claudio Sartini, statisticians from Bayer AG, for their support in developing the statistical part of the protocol; Simone Werner, of Bayer AG, for her work on the literature review and implementation of the semi-quantitative interviews; and Ellen de Waal, HARMONY communication manager, who supported the recruitment of participants.

\section{Authors' contributions}

All authors made substantive contributions to the design of the study. $\mathrm{KL}$, $\mathrm{RG}, \mathrm{RS}-\mathrm{R}, \mathrm{KH}$, and PW elaborated the study protocol in detail and the coauthors critically reviewed it. KL drafted the manuscript, and all co-authors reviewed the manuscript and approved it to be published. All authors read and approved the final manuscript. 


\section{Funding}

This work was funded through the Innovative Medicines Initiative 2 joint undertaking and listed under grant agreement 116026. This joint undertaking receives support from the European Union's Horizon 2020 research and innovation program and the EFPIA. An additional cash grant was obtained from Bayer AG for conducting the Delphi survey development. The manuscript was written and reviewed by different EFPIA members and members of the HARMONY Alliance, who were also involved in study design. Data analysis and interpretation will be performed with support from Bayer AG.

\section{Availability of data and materials}

Not applicable.

\section{Ethics approval and consent to participate}

The HARMONY Alliance is structured into eight WPs, of which WP 8 is responsible for ethical aspects of the project. Further information can be found at https://www.harmony-alliance.eu/en/work-packages/work-package8-legal-ethics-and-governance. In consultation with the WP 8 , the development and conducting of the Delphi survey were performed, and ethics approval is not required. Recruitment of participants is carried out within the HARMONY Alliance. Patient recruitment is carried out by patient umbrella organizations, partners in HARMONY. Before registration, all study participants receive information about the study, and informed consent is obtained from all participants during registration.

\section{Consent for publication}

Not applicable.

\section{Competing interests}

All authors are members of the HARMONY consortium. The authors declare that they have no other competing interests with regard to the Delphi study protocol. HCS, RG, and RSR are employed by Celgene, AbbVie, and Bayer, respectively.

\section{Author details}

${ }^{1}$ Charité University Medicine, Chariteplatz 1, Berlin 10117, Germany. ${ }^{2}$ NICE, National Institute for Health and Care Excellence, 10 Spring Gardens, London SW1A 2BU, UK. ${ }^{3}$ MRC North West Hub for Trials Methodology Research, University of Liverpool, Brownlow Hill, Liverpool L69 7ZX, UK. ${ }^{4}$ University of Cambridge, Trinity Lane, Cambridge CB2 1TN, UK. ${ }^{5}$ VU University Medical Center, De Boelelaan 1105, Amsterdam 1081 HV, Netherlands. ${ }^{6}$ Patvocates, Am Rothenanger 1b, Riemerling 85521, Germany. ${ }^{7}$ Universidad de Salamanca, Patio de Escuelas, 1, Salamanca 37008, Spain. ${ }^{8}$ Celgene International, Route de Perreux 1, Boudry CH-2017, Switzerland. ${ }^{9} \mathrm{AbbVie}, 14$ Riverwalk, Dublin D24 XN32, Ireland. ${ }^{10}$ Bayer AG, Müllerstraße 178, Berlin 13353, Germany.

Received: 24 July 2019 Accepted: 6 May 2020

Published online: 27 May 2020

\section{References}

1. Papaemmanuil E, Gerstung M, Bullinger L, Gaidzik VI, Paschka P, Roberts ND, et al. Genomic classification and prognosis in acute myeloid leukemia. N Engl J Med. 2016;374(23):2209-21.

2. Döhner H, Estey E, Grimwade D, Amadori S, Appelbaum FR, Büchner T, et al Diagnosis and management of AML in adults: 2017 ELN recommendations from an international expert panel. Blood. 2017:129(4):424-47.

3. Williamson PR, Altman DG, Bagley H, Barnes KL, Blazeby JM, Brookes ST, et al. The COMET handbook: version 1.0. Trials. 2017;18(3):280

4. Kirkham JJ, Davis K, Altman DG, Blazeby JM, Clarke M, Tunis S, et al. Core outcome set standards for development: the COS-STAD recommendations. PLoS Med. 2017:14(11):e1002447.

5. Kirkham JJ, Gorst S, Altman DG, Blazeby JM, Clarke M, Tunis S, et al. Core outcome set STAndardised protocol items: the COS-STAP statement. Trials. 2019;20:116

6. http://www.comet-initiative.org. Accessed 17 Oct 2017

7. Cheson BD, Benett JM, Kopecky KJ, Büchner T, Willman CL, Estey EH, et al. Revised recommendations of the International Working Group for diagnosis, standardization of response criteria, treatment outcomes and reporting standards for therapeutic trials in acute myeloid leukemia. J Clin Oncol. 2003;21(24):4642-9.

8. Gargon E, Gurung B, Medley N, Altman DG, Blazeby JM, Clarke M, et al. Choosing important health outcomes for comparative effectiveness research: a systematic review. PLoS One. 2014;9(6):e99111.

9. Gorst SL, Gargon E, Clarke M, Blazeby JM, Altman DG, Williamson PR Choosing important health outcomes for comparative effectiveness research: an updated review and user survey. PLoS One. 2016;11(1): e0146444.

10. Gorst SL, Gargon E, Clarke M, Smith V, Williamson PR. Choosing important health outcomes for comparative effectiveness research: an updated review and identification of gaps. PLoS One. 2016;11(12):e0168403.

11. Reeve BB, Mitchell SA, Dueck AC, Basch E, Cella D, Miller Reley C, et al. Recommended patient-reported core set of symptoms to measure in adult cancer treatment trials. J Natl Cancer Inst. 2014;106(7):dju129.

12. Buckley SA, Kirtane K, Walter RB, Lee SJ, Lyman GH. Patient reported outcomes in acute myeloid leukemia: where are we now? Blood Rev. 2017; 32(1):81-7.

13. Efficace F, Gaidano G, Lo-Coco F. Patient-reported outcomes in hematology: it is time to focus more on them in clinical trials and hematology practice? Blood. 2017;130(7):859-66.

14. Korol EE, Wang S, Johnston K, Ravandi-Kashani F, Levis M. van Nooten F. Health-related quality of life of patient with acute myeloid leukemia: a systematic literature review. Oncol Ther. 2017;5:1-16.

15. Gargon E, Crew R, Burnside G, Williamson PR. Higher number of items associated with significantly lower response rates in COS Delphi surveys. J Clin Epidemiol. 2019;108:110-20.

16. Fish R, Sanders C, Williamson PR, Renehan AG. Core outcome research measures in anal cancer (CORMAC): protocol for systematic review, qualitative interviews and Delphi survey to develop a core outcome set in anal cancer. BMJ Open. 2017;7:e018726.

\section{Publisher's Note}

Springer Nature remains neutral with regard to jurisdictional claims in published maps and institutional affiliations.
Ready to submit your research? Choose BMC and benefit from:

- fast, convenient online submission

- thorough peer review by experienced researchers in your field

- rapid publication on acceptance

- support for research data, including large and complex data types

- gold Open Access which fosters wider collaboration and increased citations

- maximum visibility for your research: over $100 \mathrm{M}$ website views per year

At BMC, research is always in progress.

Learn more biomedcentral.com/submissions 